\title{
Plasma levels of D-dimer in a 5-year-old girl with systemic juvenile idiopathic arthritis: A case report and literature review
}

\author{
QIANG XU and CHANG-SONG LIN
}

Department of Rheumatology and Immunology, The First Affiliated Hospital of Guangzhou University of Chinese Medicine, Guangzhou, Guangdong 510405, P.R. China

Received October 21, 2014; Accepted September 24, 2015

DOI: 10.3892/etm.2016.3009

\begin{abstract}
The present study reported high levels of D-dimer associated with multiple joint pain in a 5-year-old patient with systemic juvenile idiopathic arthritis (JIA). While treatment with methotrexate (MTX), hydroxychloroquine and methylprednisolone was found to reduce the D-dimer level and alleviated joint pain, the fever was not effectively controlled. Addition of etanercept to the treatment regimen from week 2 resulted in a clinical remission. Following 4-6 months of therapy, the D-dimer level of the patient returned to the normal range, and symptoms of noticeable joint pain and fever were absent. A literature search showed that the levels of D-dimer may be associated with JIA disease severity, and can serve as a prognostic biomarker for JIA treatment. In conclusion, the present study demonstrated that, while MTX therapy effectively reduced D-dimer levels, addition of etanercept to the treatment regimen was required to achieve a long-lasting remission of clinical symptoms, including fever.
\end{abstract}

\section{Introduction}

Juvenile idiopathic arthritis (JIA) is defined as a chronic inflammatory disease associated with arthritis of unknown etiology. It is a form of arthritis that is observed in childhood, occurring in 16-150 out of 100,000 children, and thus is the most common chronic rheumatic disease affecting children worldwide (1). JIA is diagnosed in cases where the age at onset is $<16$ years, the disease duration is 6 weeks or greater, and other known conditions are excluded $(2,3)$. All presentations of JIA share the common characteristic of chronic joint inflammation. Based on its pathology, JIA is classified into several categories, including systemic, polyarticular, oligoarticular, psoriatic, enthesitis-associated and undifferentiated JIA $(4,5)$.

Correspondence to: Dr Qiang Xu, Department of Rheumatology and Immunology, The First Affiliated Hospital of Guangzhou University of Chinese Medicine, 16 Jichang Road, Guangzhou, Guangdong 510405, P.R. China

E-mail: fjksg@163.com

Key words: juvenile idiopathic arthritis, D-dimer, etanercept
Although systemic JIA is the rarest form of juvenile arthritis ( $\sim 10 \%$ of all types of JIA) (6), it is the most difficult to treat and has a disproportionately high morbidity compared with the other subtypes (7). Additionally, systemic JIA frequently results in the worst outcome compared with the other subtypes, as it affects the entire body. It is generally considered that JIA severity increases with the number of joints affected. The possibility that a patient will eventually experience a total remission of symptoms decreases with increasing disease severity (8).

The present study described the typical case of a patient who was diagnosed with systemic JIA associated with notably elevated plasma levels of D-dimer. Following 6 months of medical treatment, the patient's joint swelling and pain were controlled, and the D-dimer levels were considerably decreased. The treatment and outcome of the present case were also discussed.

\section{Case report}

A 5-year-old Chinese girl presented at the Department of Rheumatology and Immunology (The First Affiliated Hospital of Guangzhou University of Chinese Medicine, Guangzhou, China) with severe pain in multiple joints in August 2013. The patient had progressive swellings at the neck, waist, elbows, wrists, knees, ankles and knuckles. The patient's medical history was normal, with up-to-date immunizations and no notable family history of autoimmune diseases, including JIA. At the first examination following admission, the patient was noted to be tired and pale, and the highest axillary temperature was $39.2^{\circ} \mathrm{C}\left(102.5^{\circ} \mathrm{F}\right)$.

The patient had felt unwell for $>2$ months, and initially presented with joint swelling and pain in the left knee. She had been previously diagnosed in a local clinic and received external medical treatment (10 days earlier, in August 2013; treatment details unknown); however, the condition was not improve noticeably. Subsequently, 12 days after treatment at the local clinic, the patient presented recurrent fever and was administered a fever-reducing medication (ibuprofen) when the fever reached $38.5^{\circ} \mathrm{C}\left(101.3^{\circ} \mathrm{F}\right)$. Following administration of antipyretic, the temperature returned to normal. However, 2 weeks before admission to our hospital, the patient experienced severe swelling and pain in the medial sides of her neck, waist, elbows, wrists, knees, ankles and knuckles. The severe 
Table I. Clinical laboratory test results.

\begin{tabular}{|c|c|c|c|c|c|c|c|}
\hline Date & WBC $\left(10^{9} / 1\right)$ & $\operatorname{HGB}(\mathrm{g} / \mathrm{l})$ & $\operatorname{PLT}\left(10^{9} / 1\right)$ & $\operatorname{ESR}(\mathrm{mm} / \mathrm{H})$ & CRP (mg/l) & $\mathrm{RF}(\mathrm{U} / \mathrm{ml})$ & D-dimer (ng/ml) \\
\hline Aug 25, 2013 & 11.71 & 77 & 471 & 75 & 128.0 & $<20$ & - \\
\hline Aug 28, 2013 & 7.05 & 87 & 509 & 90 & 56.2 & - & 10,600 \\
\hline Sep 1, 2013 & 6.31 & 83 & 460 & 93 & 26.8 & $<20$ & 7,320 \\
\hline Sep 6, 2013 & 7.28 & 89 & 580 & 60 & 9.6 & $<20$ & 2,460 \\
\hline Nov 23, 2013 & 8.40 & 129 & 328 & 5 & 1.2 & $<20$ & 717 \\
\hline Dec 21, 2013 & 6.40 & 131 & 368 & 4 & 2.2 & $<20$ & 351 \\
\hline Feb 11, 2014 & 7.80 & 132 & 378 & 3 & $<1.0$ & $<20$ & 336 \\
\hline
\end{tabular}

WBC, white blood cell count; HGB, hemoglobin; PLT, platelet count; ESR, erythrocyte sedimentation rate; CRP, C-reactive protein; RF, rheumatoid factor.
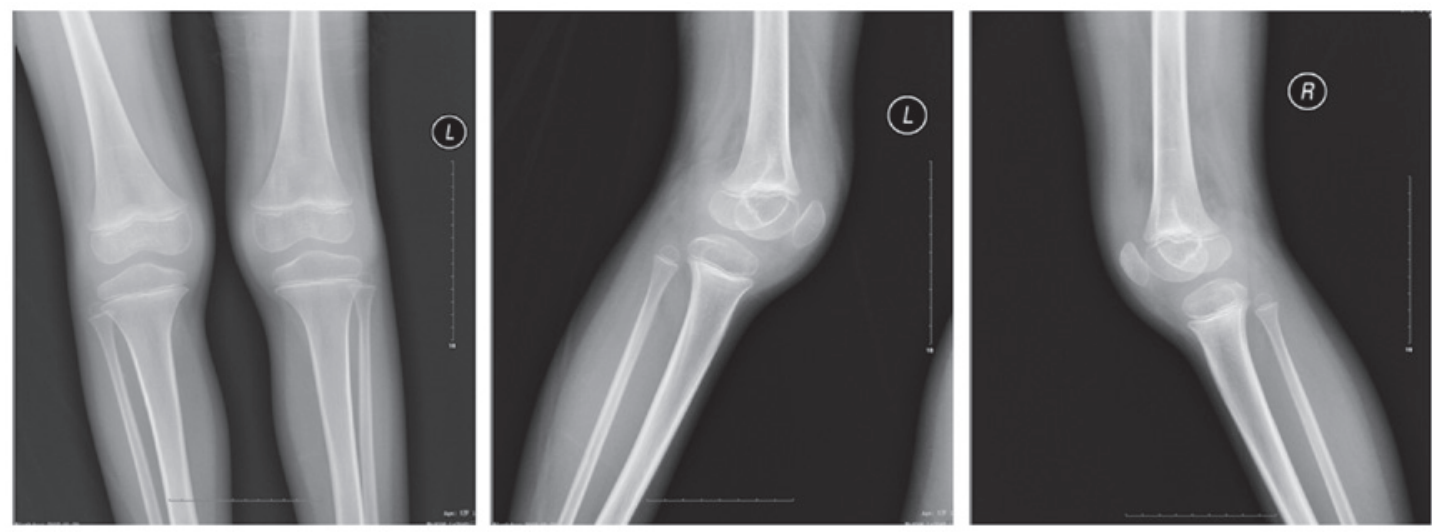

Figure 1. Medical radiography (T2-weighted) of the knees, showing swelling at the joints. L, left; R, right.

pain was accompanied by a fever, which usually occurred once daily in the evening. The patient was unable to stand or ambulate without assistance.

A physical examination revealed that the patient had no erythrasma or hemorrhagic spots on the skin (rash), and the superficial lymph nodes were palpable in the size range between $0.5 \times 0.5$ and $0.5 \times 1 \mathrm{~cm}$. No adhesions with the surrounding tissues and no tenderness were observed. In addition, the patient displayed throat congestion with blood and bilateral amygdala enlargement with degree I swelling (9), but no evidence of pyosis. Further physical examination demonstrated that the heart and lung functions were normal, while the abdomen was soft, non-tender and without evidence of abdominal masses. The liver was palpable at $1.5 \mathrm{~cm}$ below the right costal margin at the right midclavicular line and $1 \mathrm{~cm}$ below the xiphoid process, but not below the spleen rib. The patient experienced tenderness (mild to moderate) over the sternum, but not over the cervical and lumbar vertebrae. Joint tenderness and swelling involved the shoulders, elbows, wrists, bilateral metacarpophalangeal 1-5, proximal interphalangeal joints 1-5, bilateral hip, knees and ankles, while the patient presented limited range of motion in the aforementioned joints. Restricted ranges of flexion and extension were observed in the neck and lumbar regions.

Laboratory tests (results presented in Table I) performed at the initial visit of the patient in our hospital showed a white blood cell (WBC) count of 11,710/ $\mu$ l (normal range, 4-10x10\%/l), platelet count of 471,000/ $\mu 1$ (normal range, 100-300x10 $/ 1$ ), hemoglobin level of $77 \mathrm{~g} / 1$ (normal range, 120-160 g/l), C-reactive protein (CRP) level of $128 \mathrm{mg} / \mathrm{l}$ (normal range, $0-8 \mathrm{mg} / \mathrm{l}$ ), an erythrocyte sedimentation rate (ESR) of $75 \mathrm{~mm} / \mathrm{h}$ (normal range, $0-15 \mathrm{~mm} / \mathrm{h}$ ) and a noticeably elevated plasma level of D-dimer $(10,600 \mathrm{ng} / \mathrm{ml}$; normal range, 0-324 ng/ml). Tests for antinuclear antibodies (ANAs) and double-stranded DNA were negative, while the complement C3 and C4 levels and the results of an anti-extractable nuclear antigen antibody test were normal. Furthermore, the levels of anti-cyclic citrullinated peptide antibody and rheumatoid factor (RF) were found to be $<0.5 \mathrm{U} / \mathrm{ml}$ (normal range, $0-20 \mathrm{U} / \mathrm{ml}$ ) and $<20 \mathrm{U} / \mathrm{ml}$ (normal range, 0-5 U/ml), respectively, while a test for glucose phosphate isomerase was negative. The patient was suspected to suffer from systemic JIA, thus, X-ray examination was performed in order to rule out other potential causes of symptoms (such as fracture). A radiography examination revealed no abnormalities in the chest; however, the knee joint capsules of the patient were swollen (Fig. 1). Furthermore, a color Doppler ultrasound showed hepatomegaly, but no abnormal findings were observed for the gallbladder, spleen, pancreas, kidneys, ureter or urinary bladder. Echocardiography revealed no abnormalities.

Based on the JIA classification system proposed by the International League of Associations for Rheumatology, the patient was diagnosed with systemic JIA (4). The patient was treated with methotrexate (MTX; $5 \mathrm{mg}$ per week; 
Sine Pharmaceutical, Co., Ltd., Shanghai,China), hydroxychloroquine (HCQ; 100 mg, twice a day; Zhongxi Pharmaceutical Co., Ltd.,Shanghai,China) and methylprednisolone tablets (Medrol, 4 mg per day; Pfizer, Inc., New York, NY, USA) for 7 days. Low doses of folic acid (5 mg per week; Changzhou Pharmaceutical Factory, Changzhou, China) and vitamin D (chewable tablets; Xingsha Pharmaceutical Co., Ltd., Xiamen, China) were also prescribed to minimize the treatment side effects. However, although the patient's D-dimer level decreased from 10,600 to 2,460 ng/ml, the fever could not be controlled. Therefore, etanercept (12.5 mg per week, subcutaneous injection; Pfizer, Inc.) was added at the beginning of week 2. Following the administration of etanercept, clinical remission was rapidly obtained, accompanied by an absence of joint swelling and pain, and no recurrence of fever. After 6-months of treatment, the D-dimer levels, CRP levels and ESR values of the patient were considerably decreased. No adverse events occurred during the treatment.

The most recent follow-up was conduced in August 2015, at which the patient continued the treatment with MTX, HCQ and etanercept, but not with methylprednisolone. Written informed consent was obtained from the patient's guardian prior to the publication of this study.

\section{Discussion}

JIA is a form of chronic arthritis that occurs in children and has no apparent cause. Systemic JIA may initially present as a swollen knuckle, a spiking fever or an unexplained rash (4). The present study described a case of JIA in a 5-year-old Chinese girl, in whom the triggers for the disease were unknown. The patient was initially diagnosed and treated at a local clinic; however, the symptoms were not alleviated. The initial treatment details are unknown, thus it is unclear whether it exacerbated the disease. The patient presented daily fevers for $>2$ weeks and displayed lymph node enlargement. She also showed symptoms of swelling, pain and stiffness in numerous joints. No family history of HLA-B27-associated diseases was identified, and the RF of the patient was $<20 \mathrm{IU} / \mathrm{ml}$, while the ANA test gave negative results; these are characteristic features of systemic JIA as compared with other types of JIA (4). After excluding infection, leukemia, malignancy and other rheumatic diseases, the patient was diagnosed with systemic JIA.

The various forms of JIA are a heterogeneous group of disorders presenting with certain common first symptoms, including joint pain or swelling, and reddened or warm joints (10). Although systemic JIA is the least prevalent JIA subtype, it is often associated with the worst outcomes, and has disproportionately high morbidity compared with the other subtypes (11). Due to its distinct clinical and epidemiological features, systemic JIA has been recognized as a unique condition among childhood arthritis diseases (12). Systemic JIA poses a formidable challenge to pediatric rheumatologists, since prediction of its long-term clinical course and outcome based on its initial presentation and early course is difficult. In addition, there is no single definitive laboratory test for the diagnosis of systemic JIA.

Studies have shown that endothelial activation is a key component in the pathogenesis of JIA, and that the levels of biomarkers for endothelial activation are usually elevated in JIA patients with the systemic subtype $(13,14)$. Among these biomarkers, soluble intercellular adhesion molecule-1 (sICAM-1), E-selectin and D-dimer have been reported as being predictive for JIA disease activity $(15,16)$. Elevated D-dimer levels have been correlated with the elevation of sICAM-1 levels. Bloom et al found that levels of fibrin D-dimer were correlated with short-term outcomes and the response to immunomodulatory therapies in patients with systemic JIA $(13,16,17)$. A normal plasma D-dimer level is $<250 \mathrm{ng} / \mathrm{ml}$; however, in the current case, a significantly elevated level of D-dimer $(10,600 \mathrm{ng} / \mathrm{ml})$ was observed at the first examination upon admission. In addition, abnormally high levels of WBC, ESR and CRP were observed. Elevated plasma D-dimer levels typically reflect the activation of the procoagulant and fibrinolytic systems; however, the abnormal D-dimer level in the patient of the present study was much higher compared with the initial median value $(\sim 1,335 \mathrm{ng} / \mathrm{ml})$ that is commonly detected in children with thrombosis (18). Thrombosis was also excluded based on the patient's other clinical information. Bloom et al reported that, among 24 patients with systemic JIA, 23 (96\%) were found to have elevated D-dimer levels (16). D-dimer levels are correlated with fever and total leukocyte count, but not with the ESR, the duration of morning stiffness or swollen joint counts. In the current case, we observed abnormally high levels of WBC, ESR and CRP, in addition to elevated D-dimer levels.

After 1 week of treatment with MTX, HCQ and methylprednisolone, joint pain was alleviated and the patient's D-dimer level was reduced from 10,600 to $2,460 \mathrm{ng} / \mathrm{ml}$, suggesting an association of D-dimer levels with joint pain. However, the patient's fever could not be controlled, suggesting that these medications were not sufficient to alleviate all symptoms. Etanercept administration is recommended for children aged 4-17 years who have active JIA in at least five joints, and whose condition has not adequately responded to MTX treatment (11). In the present case, a clinical remission was quickly achieved after addition of etanercept to the treatment regimen from week 2 . Fever, joint swelling and pain were less severe, and the results of laboratory tests showed a continuous decline in D-dimer levels, reaching $717 \mathrm{ng} / \mathrm{ml}$ after 2 months of treatment. Recent studies conducted in adult RA patients have observed encouraging results concerning the use of MTX in combination with the new anti-tumor necrosis factor drugs, etanercept and infliximab (19). Those studies reported near normal D-dimer levels ( 330 ng/ml) following 4-6 months of treatment. This treatment duration is consistent with that expected for MTX therapy, where the maximum therapeutic effect is usually achieved 4-6 months after initiation of the treatment (20). The reduction in D-dimer levels was correlated with the patient's level of disease activity, and was associated with reductions in ESR and CRP levels. During the constant follow-up, no signs of complications were observed.

In the case reported in the present study, reduced D-dimer levels were accompanied by rapid clinical remission, as well as reduction and later absence of joint swelling and pain. At present, there are no guidelines for making clinical decisions regarding for how long MTX treatment should be continued following disease remission. Currently, the patient of the present study continues to be treated with MTX, HCQ and 
etanercept, but not methylprednisolone. At the time of the most recent visit (August 2015), the patient presented no joint swelling, joint pain or incidence of recurrent fever, and continued to receive treatment with MTX, HCQ and etanercept, but not with methylprednisolone. Due to the high relapse rate in JIA patients following MTX withdrawal, a weekly regimen of MTX, HCQ and etanercept using the previous dosages was continued for an additional 6 months, until a sustained remission was achieved.

In conclusion, the present study reported the case of a 5-year-old girl who suffered from swelling and pain at multiple joints. The patient was diagnosed with systemic JIA and was treated with a combination therapy. Disease remission accompanied by decreased plasma D-dimer levels was observed after 6 months of treatment. In future studies, D-dimer may be used to evaluate the degree of disease activity in JIA patients.

\section{References}

1. Foster H, Rapley T and May C: Juvenile idiopathic arthritis: Improved outcome requires improved access to care. Rheumatology (Oxford) 49: 401-403, 2010.

2. Prakken B, Albani S and Martini A: Juvenile idiopathic arthritis. Lancet 377: 2138-2149, 2011.

3. Espinosa M and Gottlieb BS: Juvenile Idiopathic Arthritis. Pediatr Rev 33: 303-313, 2012.

4. Petty RE, Southwood TR, Manners P, Baum J, Glass DN, Goldenberg J, He X, Maldonado-Cocco J, Orozco-Alcala J, Prieur AM, et al: International League of Associations for Rheumatology classification of Juvenile Idiopathic Arthritis: Second revision, Edmonton, 2001. J Rheumatol 31: 390-392, 2004

5. Kahn P: Juvenile idiopathic arthritis: An update for the clinician. Bull NYU Hosp Jt Dis 70: 152-166, 2012.

6. Schneider R and Laxer RM: Systemic onset juvenile rheumatoid arthritis. Bailliere's Clin Rheumatol 12: 245-271, 1998.

7. Wallace CA and Levinson JE: Juvenile rheumatoid arthritis: Outcome and treatment for the 1990s. Rheum Dis Clin North Am 17: 891-905, 1991.

8. Prince FH, Otten MH and van Suijlekom-Smit LW: Diagnosis and management of juvenile idiopathic arthritis. BMJ 341 c6434, 2010 .
9. Watson C, Nielsen SL, Cobb C, Burgerman R and Williamson B. Pathological grading system for hippocampal sclerosis: Correlation with magnetic resonance imaging-based volume measurements of the hippocampus. J Epilepsy 9: 56-64, 1996.

10. Martini A: Are the number of joints involved or the presence of psoriasis still useful tools to identify homogeneous disease entities in juvenile idiopathic arthritis? J Rheumatol 30: 1900-1903, 2003

11. Quartier P, Taupin P, Bourdeaut F, Lemelle I, Pillet P, Bost M, Sibilia J, Koné-Paut I, Gandon-Laloum S, LeBideau M, et al: Efficacy of etanercept for the treatment of juvenile idiopathic arthritis according to the onset type. Arthritis Rheum 48: 1093-1101, 2003.

12. Mellins ED, Macaubas C and Grom AA: Pathogenesis of systemic juvenile idiopathic arthritis: Some answers, more questions. Nat Rev Rheumatol 7: 416-426, 2011.

13. Bloom BJ, Nelson SM, Eisenberg D and Alario AJ: Soluble intercellular adhesion molecule-1 and E-selectin as markers of disease activity and endothelial activation in juvenile idiopathic arthritis. J Rheumatol 32: 366-372, 2005.

14. Turhan H, Erbay AR, Yasar AS, Aksoy Y, Bicer A, Yetkin G and Yetkin E: Plasma soluble adhesion molecules; intercellular adhesion molecule-1, vascular cell adhesion molecule-1 and E-selectin levels in patients with isolated coronary artery ectasia. Coron Artery Dis 16: 45-50, 2005.

15. Dolezalova P, Telekesová P, Nemcová D and Hoza J: Soluble adhesion molecules ICAM-1 and E-selectin in juvenile arthritis: Clinical and laboratory correlations. Clin Exp Rheumatol 20: 249-254, 2002.

16. Bloom BJ, Tucker LB, Miller LC and Schaller JG: Fibrin D-dimer as a marker of disease activity in systemic onset juvenile rheumatoid arthritis. J Rheumatol 25: 1620-1625, 1998.

17. Bloom BJ, Alario AJ and Miller LC: Persistent elevation of fibrin D-dimer predicts longterm outcome in systemic juvenile idiopathic arthritis. J Rheumatol 36: 422-426, 2009.

18. Goldenberg NA, Knapp-Clevenger R and Manco-Johnson MJ; Mountain States Regional Thrombophilia Group: Elevated plasma factor VIII and D-dimer levels as predictors of poor outcomes of thrombosis in children. N Engl J Med 351: 1081-1088, 2004.

19. Keyser FD: Choice of Biologic Therapy for Patients with Rheumatoid Arthritis: The Infection Perspective. Curr Rheumatol Rev 7: 77-87, 2011.

20. American College of Rheumatology Subcommittee on Rheumatoid Arthritis Guidelines: Guidelines for the management of rheumatoid arthritis: 2002 Update. Arthritis Rheum 46: $328-346,2002$. 